\title{
Ecological and Physiological Assessment of Animal Anesthesia Methods
}

\author{
K A Sidorova ${ }^{1}$, O A Dragich ${ }^{1,2}$, S A Ermolina ${ }^{3}$, O V Kochetova ${ }^{4}$, and \\ O A Balabanova ${ }^{1}$ \\ ${ }^{1}$ Northern Trans-Ural State Agricultural University, Tyumen, Russia \\ ${ }^{2}$ Tyumen Industrial University, Tyumen, Russia \\ ${ }^{3}$ Vyatka state agricultural Academy, Kirov, Russia \\ ${ }^{4}$ Perm Institute of the Federal penitentiary service of Russia, Perm, Russia
}

\section{Abstract}

The use of pharmaceuticals is indispensable in everyday medical practice. The patient's life sometimes depends on the right dosages and the chosen method of administration of the drug, therefore, for the successful provision of assistance to the animal, the specialist should become familiar with pharmacokinetics and pharmacodynamics of the drugs used to prevent undesirable side effects and to reduce the risk of adverse outcome of the disease. Currently, many veterinary enterprises have been opened

Corresponding Author:

K A Sidorova

IBVM.veterinarya@yandex.ru

Received: 25 October 2019

Accepted: 15 November 2019

Published: 25 November 2019

Publishing services provided by Knowledge E

(c) K A Sidorova et al. This article is distributed under the terms of the Creative Commons

Attribution License, which

permits unrestricted use and redistribution provided that the original author and source are credited.

Selection and Peer-review under the responsibility of the AgroSMART 2019 Conference Committee. in Russia, a considerable number of them are private veterinary clinics, where daily surgical interventions with the use of anesthetics are carried out. Depending on the equipment they use different methods of anesthesia of animals -- inhalation, non-inhalation or a combined method. The article deals with pharmacokinetic factors in the field of veterinary anesthesiology, its peculiarities associated with different methods of introduction of different groups of anesthetics, which have a similar effect on the body when injected into anesthesia. Moreover, depending on the method of introduction, their absorption into the body varies, the depth of anesthesia changes and the time of exit from it is different.

Keywords: anaesthesia, method, clinic, condition, animals, anesthetics, concentration, surgery.

\section{Introduction}

Physicians cannot do without the use of medicines. The patient's life sometimes depends on the right dosages and the chosen method of administration of the drug, therefore, for the successful provision of care to the animal, the specialist should become familiar with pharmacokinetics and pharmacodynamics of the drugs used to prevent undesirable side effects and to reduce the risk of adverse outcome of the disease.

Nowadays many veterinary enterprises have been opened, a considerable number of them are private veterinary clinics, which perform daily surgical interventions with 
the use of anesthetics. Depending on the equipment they use different methods of anesthesia of animals -- inhalation, non-inhalation, or a combined method.

Therefore, the purpose of the research is the scientific substantiation of the use of inhalation and non-inhalation anesthesia in veterinary surgery.

\section{Material and Methods of Research}

The work was carried out in the conditions of the Department of Anatomy and Physiology of the Institute of Biotechnology and Veterinary Medicine of the Federal State Budgetary Institution of Higher Education "State Agrarian University of the Northern Urals" and in the veterinary clinics of Tyumen.

The study focused on non-inhalation anesthetics such as zolethyl, propofol and inhalation anesthetics such as isoflurane and nitrous oxide. The object for the study of pharmacokinetic properties of the drugs were cats and dogs of different breeds and pedigree, of different sex and age, who were prescribed general anesthesia for surgical interventions. A total of 19 cats and 22 dogs were examined for general anesthesia. During the operation, control over the animal's entrance to anesthesia, monitoring of his heart rate, blood pressure, saturation during the operation under general anesthesia and observation of the exit from general anesthesia in the patient.

Animals that underwent Doppler examination of renal hemodynamics with the help of ultrasound machine were selected for the analysis of the PC mechanism in noninhalation anesthesia.

\section{The Results of the Study and Their Discussion}

General anesthesia is performed in almost all veterinary clinics with non-inhalation anesthesia and only in some clinics inhalation anesthesia is used during surgical procedures.

This is due to the fact that for general anesthesia inhalation method requires a specially equipped room with ventilation, with equipment for supplying inhalation anesthesia and monitoring the condition of the animal. It is necessary to follow the rules of storage of inhalation anesthetics, as some of them are explosive and even inhalation of fumes in the operating room during general anesthesia with open or semi-open circuit is considered unsafe for workers. An anesthesiologist with expertise in polynarcotics, MinorVet, and other anesthesia equipment should be available at the workplace to provide an anesthesia, knowing the contours of the anesthetic, and monitoring the 
oxygen supply rate to the patient's respiratory tract through the mask and endotracheal tube..

Nowadays three inhalation anesthetics are widely used in clinics: isoflurane, sevoflurane and nitrous oxide. A primitive dropper is replaced by evaporators. These are precise, calibrated devices capable of ensuring the concentration of anesthetic vapors with an accuracy of up to a tenth of a percent, regardless of air temperature.

The device is designed so that it controls itself by comparing the set and actual ventilation parameters, and monitors the compliance of the task execution. When exceeding the limits of the admissible device will inform about it by an audible signal and will display the necessary information on the screen. The anesthetic monitor controls the patient's condition. It is able to monitor many parameters of the body and the composition of the inhaled and exhaled gas mixture.

The monitor monitors the most important parameters: electrocardiogram, blood pressure, oxygen saturation (pulse oximetry) and carbon dioxide content in exhaled air (capnography).

Close to ideal anesthesia in veterinary practice is considered to be combined anesthesia, which includes the use of non-inhalation and inhalation anesthetics, as anesthetics of both groups complement each other. Thanks to the combined use of lower dosages of anesthetics, but the animal will recover from the general anesthesia for a longer period of time than if one of the two types of anesthesia is used separately.

Therefore, most veterinary clinics use non-inhalation anesthesia, also taking into account the individual characteristics of the patients. In intravenous anesthesia, the cat family wakes up quickly in general anesthesia with zoletil. This is due to the rapid elimination of anesthetics, this effect can be slowed down, increasing the dosage, but do not forget to observe the overall condition of the patient, in addition to prolong and facilitate the course of anesthesia in the animal can be premedication before surgery: xylazine, atropine sulfate, etc. In case of inhalation anesthesia it is also possible to use premedication to create a similar effect.

Depending on the condition of the sick animal, taking into account the age, gender, weight, presence or absence of concomitant diseases, allergic reactions, or intolerance to drugs, an anesthesiologist selects what is most effective and safe for the patient.

When calculating the pharmacokinetic parameters of intravenous anesthesia and inhalation anesthesia, different formulas are used, which have different parameters and values, as it is influenced by the methods of administration of anesthetics. This is the main difference between the single-phase and two-phase pharmacokinetic model. 
But at least one of the most important indicators converges by measurement standards, it is physiological clearance, an indicator that determines the ability of the body to remove drugs in their pure form and its metabolites from the body. With the help of this indicator it is revealed how the rate of removal of the drug from the body affects the dependence of the concentration of medicinal products on time. Data on the clearance of anesthetized animals vary greatly depending on the type of anesthetic and route of administration. Figure 1 shows the clearance rates for inhalation and intravenous anesthesia.

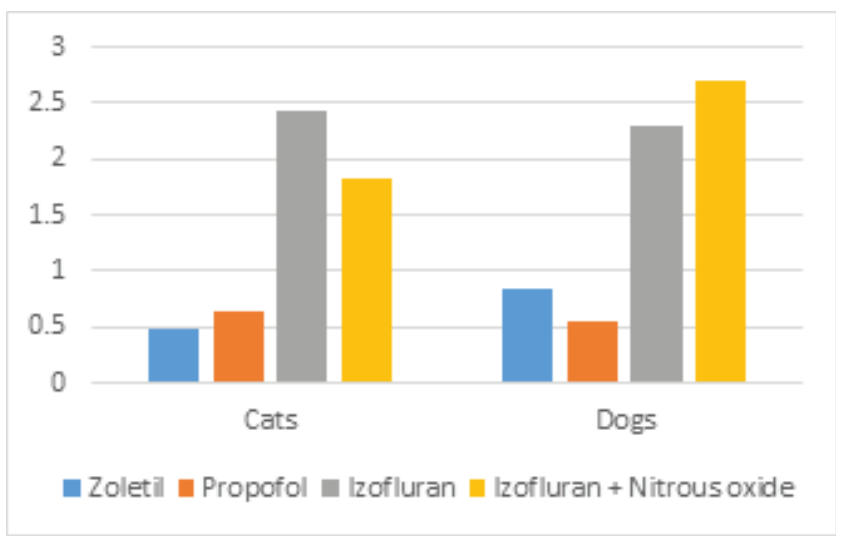

Figure 1: Ratio of average physiological clearance values for inhalation and non-inhalation anesthesia.

According to the data obtained, the average value of elimination from the body in all cases is higher in inhalation anesthesia. The difference in cats between non-inhalation anesthetic zoletil and inhalation isoflurane was $1.94 \mathrm{ml} / \mathrm{h}$, the ratio of average values of physiological clearance of zoletil and combined inhalation anesthesia isoflurane with nitrous oxide showed a difference of $1.33 \mathrm{ml} / \mathrm{h}$. In intravenous anesthesia of cats with propofol, the difference between it and inhalation anesthetic isoflurane was $1.78 \mathrm{ml} / \mathrm{h}$. When comparing the mean values of elimination from the organism of propofol hypnotic and inhalation combination of isoflurane and nitrous oxide, the difference was $1.17 \mathrm{ml} / \mathrm{h}$.

The average physiological clearance in dogs using zoletil is $1.46 \mathrm{ml} / \mathrm{h}$ lower than in inhaled mononarcoseisoflurane, the difference between the elimination of zoletil from the body and the combined inhalation mixture of isoflurane and cheerful gas was 1.86 $\mathrm{ml} / \mathrm{h}$. When using intravenous propofol anesthetic compared to isoflurane, the difference in isoflurane purification is $1.75 \mathrm{ml} / \mathrm{h}$ higher. At the ratio of non-inhalation anesthetics of propofol and combination of inhalation anesthesia from isoflurane and nitrous oxide, the elimination is higher in the last mixture by $2.15 \mathrm{ml} / \mathrm{h}$.

We can conclude that the fastest physiological clearance in cats with isoflurane inhalation anesthesia and in dogs with combined isoflurane and nitrous oxide inhalation 
anesthesia is the fastest. This can be explained by the fact that almost all inhalation mixture leaves the body through the respiratory tract in its pure form, while non-inhalation anesthetics are also liver and kidney clearance, with the formation of metabolites, which after general anesthesia circulate for some time in the body and are released mostly from the body of animals with urine.

The range of drug concentrations cannot be determined as precisely as possible during clinical trials, because each patient needs an individual selection of dosing modes. It should also be taken into account that if the average concentration of this type of animals is within the set average range, it cannot mean that the desired anesthesiological effect is achieved. The therapeutic range of anesthetics cannot be considered dogmatically due to the interindividual variability of pharmacokinetic parameters. That is why average values of anesthetic concentration ranges in the organism can be considered only as approximate reference points, relying entirely on the obtained data, without forgetting about individual factors. It is impossible to accurately predict the variability of such pharmacokinetic processes as absorption, distribution and excretion of the drug from the patient's body.

\section{Conclusion}

Nowadays, the tasks of anesthesiology go beyond the elimination of pain sensitivity during surgical interventions. The current work of an anesthesiologist includes "access to the vascular channel", selection of means to replenish the volume of circulating blood, selection and use of premedication protocols and other aspects of anesthesiology.

The efficacy of anesthetic protection can be judged by the fact that there are no contraindications for anesthesia in our time. For a sick animal in any condition and in any concomitant diseases, you can always choose an effective and safe anesthesia. In emergency situations, one thing is important: the sooner the patient falls into the hands of an anesthesiologist, the more chances he has to survive.

Anesthesiologists should, whenever possible, justify the prescription of the drug taking into account the reaction of a particular patient, which includes an assessment of both pharmacokinetic and pharmacodynamic characteristics of the drug.

Pharmacokinetic inhalation anesthesia is a two-phase pharmacokinetic model, which is due to the fact that the anesthetic first gets into the respiratory tract and only after hitting the alveoli penetrates into blood vessels, where it is already spread throughout the body. 
Intravenous anesthesia has a single-phase pharmacokinetic model, which is characterized by the instant flow of drugs into the bloodstream, and therefore the reaction to the anesthetic occurs instantly.

Volatile anesthetics used nowadays are safe, they do not undergo any changes in the organism, so there is no synthesis of products harmful or dangerous to organs and systems. Means for intravenous anesthesia are harmless, only a more prolonged exit from anesthesia is noticeable than after inhalation anesthesia. When choosing anesthesia, first of all, it is necessary to proceed from the fact which anesthetic is more suitable for this animal, to take into account the individual peculiarities of the organism, the type of surgical intervention. Pharmacokinetic parameters of anesthetics depend on the peculiarities of absorption of drug molecules from the place of administration, on the nature of their distribution in organs and tissues and on the method of anesthetics excretion from the body.

When comparing inhalation anesthesia with intravenous anesthesia can be concluded:

1. In terms of equipment, not every veterinary clinic will be able to use inhalation anesthesia, so that in most clinics, still non-inhalation anesthesia is in the first place because of the ease of implementation.

2. According to pharmacokinetic factors, the volume of anesthetic distribution is higher in a single-chamber pharmacokinetic model, because intravenous anesthesia enters the bloodstream directly. In a two-phase pharmacokinetic model, inhalation anesthetics first enter the respiratory tract and only then are absorbed into the bloodstream.

3. Physiological clearance, the indicator characterizing the cleansing of the body of drugs, occurs in inhalation anesthetics much faster, because most of them are removed through the inhalation pathways.

Based on the above, it can be noted that, although both types of general anesthesia studied differ in terms of pharmacokinetic parameters, but both of them are irreplaceable in veterinary practice, and yet, it should not be forgotten that any type of anesthesia is a risk to the life of the animal.

\section{References}

[1] Sintiurev, O.K., Glazunova, L.A., Plakhotnik, A.V. et al. (2019). Effectiveness of Surgical. Treatment of The Left-Sided Displacement of The Abomasum In Cows And First Heifers In Trans-Urals. Research Journal of Pharmaceutical, Biological and Chemical Sciences, no. 10(1), pp. 2136--2140. 
[2] Skipin, L., Gaevaya, E., Zaharova, E., Petukhova, V., Sidorova, K. (2016). Biogeochemistry of Heavy Metals in Trophic Chain in Terms of the South of Tumen Region. Original Research Article Procedia Engineering, vol. 165, pp. 860--868.

[3] Chasovshchikova, M.A. et al. (2017). Relationship between the genetic variants of kappa-casein and prolactin and the productive-biological characteristics of cows of the black-motley breed. Journal of Pharmaceutical Sciences and Research, vol. 9(7), pp. 1038--1044.

[4] Glazunov, Y.V., Glazunova, L.A. (2018). Phenology of pasture ticks in the Trans-Urals. Scopus Indian Veterinary Journal, vol. 95, no. 1, pp. 19--22.

[5] Glazunova, L.A., Glazunov, Yu.V., Ergashev, A.A. (2018). Ecological-epizootical situation on telasiosis among large cattle in Northern Ural region. Research Journal of Pharmaceutical, Biological and Chemical Sciences, vol. 9, no. 4, pp. 1687--1693. 